\title{
Interferometric inverse synthetic aperture radar experiment using an interferometric linear frequency modulated continuous wave millimetre-wave radar
}

\author{
D. Felguera-Martín ${ }^{1} \quad$ J.-T. González-Partida ${ }^{1} \quad$ P. Almorox-González ${ }^{1} \quad$ M. Burgos-García ${ }^{1}$ \\ B.-P. Dorta-Naranjo ${ }^{2}$
}

${ }^{7}$ Grupo de Microondas y Radar. Departamento de Señales, Sistemas y Radiocomunicaciones, Universidad Politécnica de Madrid., Ciudad Universitaria s/n, 28040 Madrid, Spain

${ }^{2}$ Departamento de Señales y Comunicaciones, Universidad de Las Palmas de Gran Canaria, Campus Universitario de Tafira, 35017 Las Palmas de Gran Canaria, Spain

E-mail: felguera@gmr.ssr.upm.es

\begin{abstract}
An interferometric linear frequency modulated continuous wave (LFMCW) millimetre-wave radar is presented, along with the results of an experiment conducted to study the feasibility of using it in a future millimetre-wave interferometric inverse synthetic aperture radar (InISAR) system. First, a description of the radar is given. Then, the signal processing chain is described, with special attention to the phase unwrapping technique. The interferometric phase is obtained by unwrapping the prominent target's phase in each antenna using a sliding frame processing technique. Cell migration issues in this method are also addressed. Simulations were carried out to illustrate and assess the processing chain and to show the effects of multipath echoes on the height measurement. In the real experiment, the range, speed and height of a moving target were tracked over consecutive inverse synthetic aperture radar (ISAR) image frames, verifying the performance of the whole system.
\end{abstract}

\section{Introduction}

The millimetre-wave band has several advantages that are making millimetre-wave radars more popular as the technology becomes more affordable. Millimetre-wave technology reduces the weight and size of radar circuits and antennas. This miniaturisation allows for the design of portable radar systems, which are especially important in interferometric systems, as these systems require at least two antennas and two receivers. Furthermore, the millimetre-wave band provides a large amount of bandwidth that can be transmitted more easily than other bands, as the relative bandwidth is low. This leads to high range resolution, allowing the discrimination of several targets in close proximity. Thus, in inverse synthetic aperture radar (ISAR) applications, millimetre-wave technologies can achieve better discrimination of the prominent scatters that form an image of a complex target. Also, the measurement of polarisation is especially useful in the millimetre-wave band. The size of targets relative to the operational wavelength means that considerable polarisation information is available from the target, which is especially important in target recognition applications.

In recent years, several authors have reported on the fundamentals, algorithms and applications of interferometric ISAR (InISAR) processing [1-14]. A three-dimensional image of a moving target can be obtained using this technique. Two antennas are used to generate a pair of ISAR images, whose phases are combined to obtain the height of the target scatters. Reported applications of InISAR are target scattering diagnosis [1-5], motion modelling $[6,7]$, moving target three-dimensional imaging [8-10], automatic aircraft landing [11], threat detection [12] and target recognition $[13,14]$.

However, use of the millimetre-wave band also presents some disadvantages. The peak power provided by commercial amplifiers in the millimetre-wave band is low. A continuous wave (CW) configuration can be used to overcome this drawback. Transmission of $\mathrm{CW}$ signals increases the mean transmitted power and therefore the maximum operative range [15]. Another problem of the use of millimetre-waves for interferometric applications is that the phase measurement will become ambiguous earlier because of the shorter wavelength, making the phase unwrapping process more difficult.

The main problems of InISAR processing are phase unwrapping, misregistration and phase scintillation [16]. The wrapped phase can be exactly unwrapped if the phase difference between adjacent pixels is less than $\pi$ everywhere in the image. This condition may be violated by noise, shadows, overlay, phase discontinuities and so on, making phase unwrapping a difficult task [17]. Phase unwrapping and misregistration problems have been widely studied in synthetic aperture radar interferometry (InSAR); 
these studies have produced a number of different algorithms $[16,17]$. However, real ISAR images of man-made targets do not always contain continuous structures. They usually consist of a set of isolated prominent scatters, such as the tail, fuselage and wings of an aircraft [11]. Thus, the phase unwrapping techniques developed for InSAR cannot be directly applied in InISAR processing. The impact of target glint can be reduced by increasing the radar resolution. As the resolution increases, it is less likely that different scatters will fall into the same resolution cell. However, echoes of the same target with different propagation paths will remain, causing phase scintillation.

In the present study, an interferometric linear frequency modulated continuous wave (LFMCW) radar is presented, along with the results of an experiment conducted to study the feasibility of using this radar in a future millimetre-wave InISAR system. This system will profit from the advantages of both the millimetre-wave band and the InISAR technique, in all of the above applications.

This article is arranged as follows: in Section 2, a description of the radar is given. In Section 3, the signal processing chain is described, with special attention to the phase unwrapping technique. Section 4 presents simulations to illustrate and assess the processing chain and to show the influence of multipath echoes in the height measurement. Finally, Section 5 presents a real experiment in which the range, velocity and height of a moving target are tracked over consecutive ISAR image frames.

\section{System description}

The sensor used for the InISAR experiment is the interferometric evolution of the portable high-resolution millimetre-wave LFMCW radar previously described [18]. This evolution was possible because of the initial modular design of the radar. The former radar was implemented with four basic subsystems or building blocks: the signal generation subsystem, the transmitter subsystem, the receiver subsystem and the intermediate frequency (IF) subsystem. In the present interferometric configuration the same building blocks were used, but the receiver and IF blocks were duplicated for the additional receiving antenna. Fig. 1 shows the block diagram of this interferometric configuration. As shown in the diagram, the sensor is a bistatic LFMCW interferometric radar formed by a signal generator, a transmitter block connected to the central antenna and two identical receiver chains formed by a receiving and $\mathrm{IF}$ block connected to the receiving antennas. All of the building blocks have been described in detail [18]. However, in the present configuration the IF blocks were replaced by low-pass filters, with cut-off frequencies at $4.5 \mathrm{MHz}$. In addition to these building blocks, a computer and a data acquisition card with two channels were used to coherently sample and process the data. It is important to point out that both receiver chains must be identical to prevent errors in the interferometric phase and the path from the antenna to the receiver must be as similar as possible. In Fig. 2, the symmetric configuration of the antennas and the waveguides is shown.

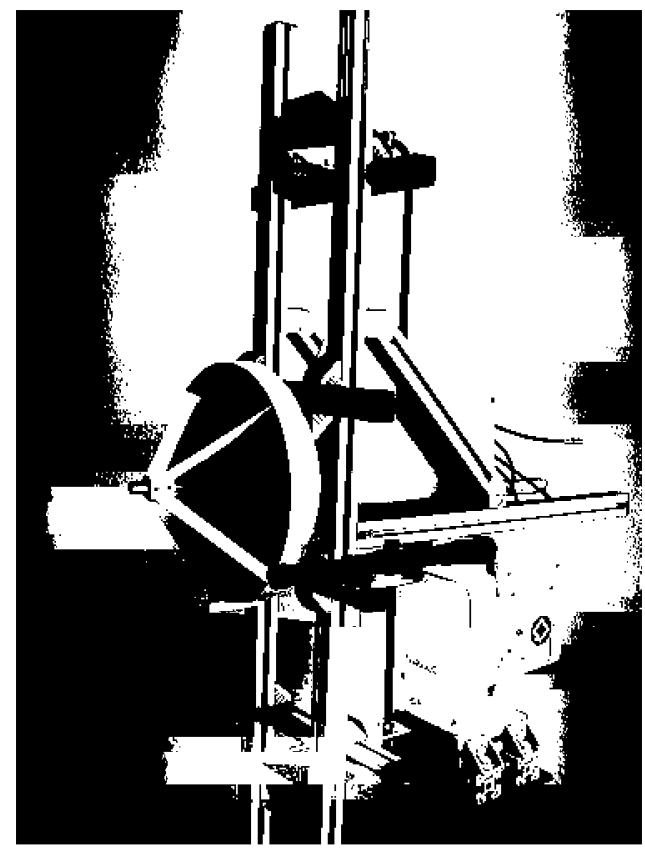

Fig. 2 Interferometric bistatic LFMCW radar demonstrator image

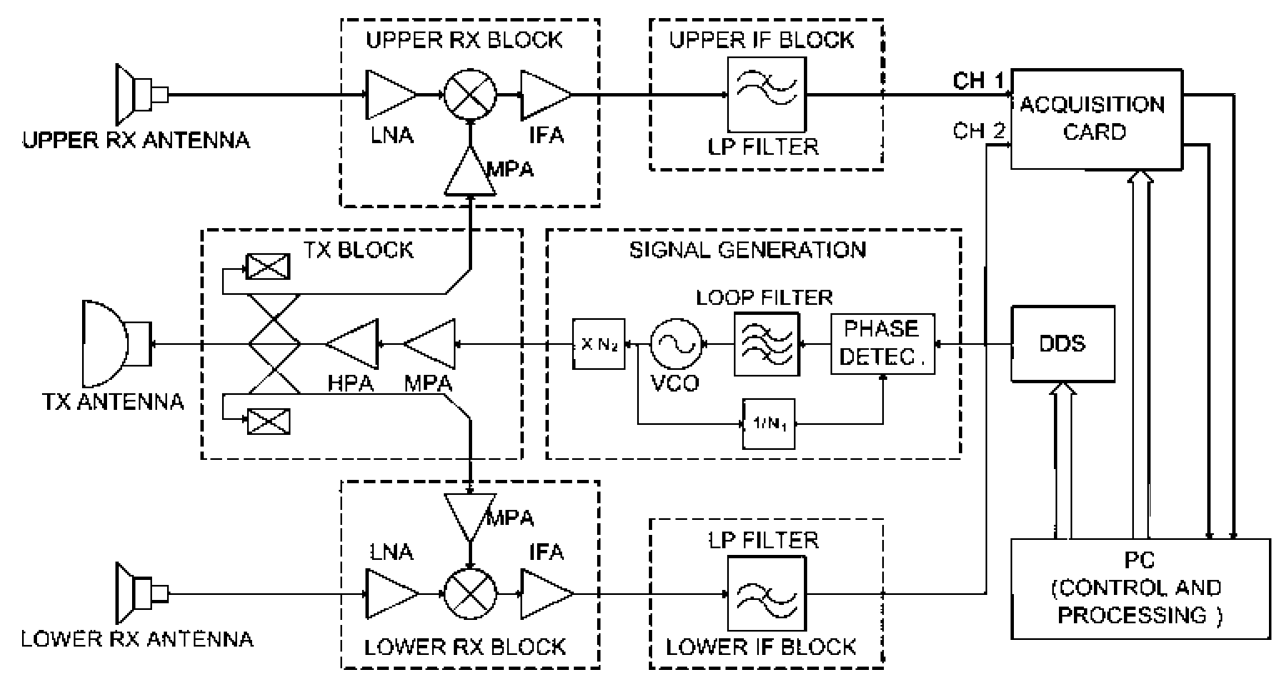

Fig. 1 Interferometric bistatic LFMCW radar demonstrator block diagram 


\section{Processing chain}

\subsection{Imaging geometry}

The imaging geometry is shown in Fig. 3. The $x$ - and $y$-axis represent the cross-range and range directions, respectively, and the $z$-axis represents the height direction. The transmitting antenna is located at $O$, and the receiving antennas are at $U$ and $L$, with coordinates $\left(x_{O}, y_{O}, z_{O}\right)$, $\left(x_{U}, y_{U}, z_{U}\right)$ and $\left(x_{L}, y_{L}, z_{L}\right)$, respectively. The target is located at $T$, with coordinates $\left(x_{T}, y_{T}, z_{T}\right)$. The distances from the antennas to the target are $r_{O}, r_{U}$ and $r_{L}$. The baseline is the distance between the receiving antennas and will be denoted by $B$. The inclination of the baseline is denoted by $\alpha$, and the elevation angle of the target as viewed from the antenna located at $L$ is called $\theta$.

Considering the geometry in Fig. 3 and trigonometric manipulations, expression (3) is obtained, which gives the height of the target as a function of $\sin (\alpha-\theta)$

$$
z_{T}=z_{L}-r_{L} \cos (\theta)
$$

$$
\begin{aligned}
\cos (\theta)= & \cos (\alpha-(\alpha-\theta)) \\
= & \cos (\alpha) \cos (\alpha-\theta)+\sin (\alpha) \sin (\alpha-\theta) \\
= & \cos (\alpha) \sqrt{1-\sin ^{2}(\alpha-\theta)}+\sin (\alpha) \sin (\alpha-\theta) \\
z_{T}= & z_{L}-r_{L} \\
& \times\left\{\cos (\alpha) \sqrt{1-\sin ^{2}(\alpha-\theta)}+\sin (\alpha) \sin (\alpha-\theta)\right\}
\end{aligned}
$$

In order to obtain the value of $\sin (\alpha-\theta)$ in (5), the law of cosines is applied to the UTL triangle

$$
\begin{aligned}
r_{U}^{2} & =B^{2}+r_{L}^{2}-2 r_{L} B \cos (\gamma) \\
& =B^{2}+r_{L}^{2}+2 r_{L} B \sin (\alpha-\theta) \\
\sin (\alpha-\theta) & =\frac{r_{U}^{2}-r_{L}^{2}-B^{2}}{2 r_{L} B} \\
& =\frac{\left(r_{L}+\Delta r\right)^{2}-r_{L}^{2}-B^{2}}{2 r_{L} B}
\end{aligned}
$$

Using (5) and (3), the height of the target as a function of the path difference $\Delta r$ can be obtained. As in any interferometric

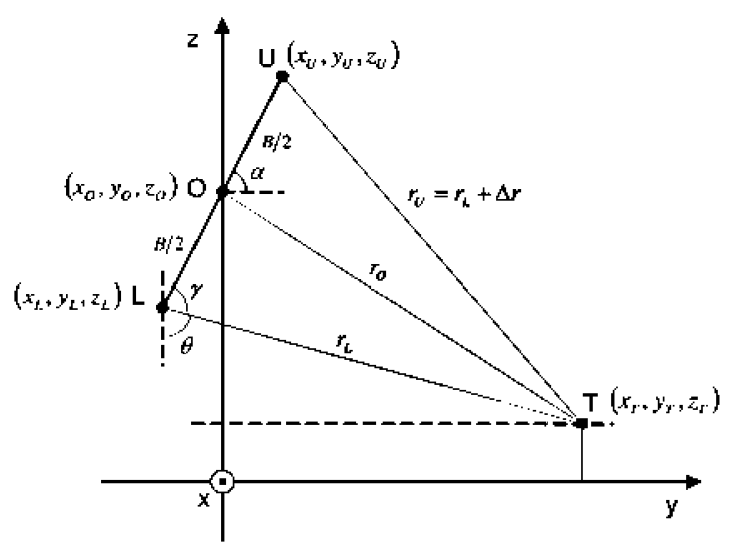

Fig. 3 Imaging geometry application, the path difference is a function of the phase difference between the receiving antennas. This phase difference is called the interferometric phase and is denoted by $\varphi_{\mathrm{I}}$. Using the interferometric phase, the path difference can be obtained with better accuracy than by using the resolution cells in which the target was detected. For bistatic radars, the phase difference is given by

$$
\begin{gathered}
\varphi_{\mathrm{I}}=\varphi_{L}-\varphi_{U} \\
=-\frac{2 \pi}{\lambda}\left(r_{O}+r_{L}\right)+\frac{2 \pi}{\lambda}\left(r_{O}+r_{U}\right) \\
=-\frac{2 \pi}{\lambda}\left(r_{L}-r_{U}\right) \\
\Delta r=r_{U}-r_{L} \\
=\frac{\lambda}{2 \pi} \varphi_{\mathrm{I}}
\end{gathered}
$$

Finally, by substituting (7) into (5) and using the result in (3), the height of the target as a function of the interferometric phase is obtained. It is worthy to point out that no approximations are made in this derivation when the target is in the azimuth pointing direction, which is very important in short-range applications where the assumption of parallel rays cannot be made.

The expression relating the height of the target to the interferometric phase is highly non-linear, and it is important to note that the absolute interferometric phase $\varphi_{\mathrm{I}}$ must be used in (7) to obtain a correct estimate of the height. However, it is only possible to measure the wrapped interferometric phase $\psi_{\mathrm{I}}$ which is related to $\varphi_{\mathrm{I}}$ by

$$
\varphi_{\mathrm{I}}=\psi_{\mathrm{I}}+2 \pi n \quad(n \in N)
$$

The absolute phase difference can be obtained by phase unwrapping or by using an unambiguous extra interferometric pair for the distance of interest. The use of extra antennas is the easier way to obtain the unwrapped phase $[6,8,9]$. However, the increased size, weight and cost of the system because of the extra antennas and receiving channels present a major drawback.

\subsection{Signal processing}

The signal processing chain is composed of three main stages. The first is the range processing stage, which obtains the range profile of each ramp. A hamming weighting window is applied prior to the range fast Fourier transform (FFT). The second stage is a sliding frame processing technique, which obtains a range-Doppler ISAR image for each set of $N$ consecutive ramps with a displacement of $M$ ramps from frame to frame. A similar processing technique is applied in [7] to a range-Doppler correlation tracker and in [10] to find the wrapped phase difference between two consecutive frames of the same antenna. A $60 \mathrm{~dB}$ Chebyshev weighting window is used in the Doppler dimension, and a simple clutter canceller is applied to reduce the clutter interference before the Doppler FFT. A set of interferometric pairs of ISAR images corresponding to each receiving antenna is obtained in the first two stages. The third stage is the interferometric processing. In this stage, the height information of the target is obtained by using the phase information in each pair of ISAR images. As shown in Section 3.1, the absolute interferometric phase must be 
obtained from the wrapped interferometric phase. As previously stated, real ISAR images of man-made targets do not present continuous structures and usually consist of a set of prominent isolated scatters. Thus, the phase unwrapping techniques developed for InSAR cannot be directly applied in InISAR processing.

The method proposed in this study for obtaining the absolute interferometric phase $\varphi_{\mathrm{I}}(n)$ is to track the phase information of the prominent scatters from frame to frame in each image pair. Then, the evolution of the wrapped phase of the scatter of each antenna is unwrapped before combining them to form the interferometric unwrapped phase. Knowing the wrapped phase of the scatter of both antennas for each frame, $\psi_{L}(n)$ and $\psi_{U}(n)$, and using the unwrapping operator $W^{-1}\{\cdot\}$, we can obtain the unwrapped interferometric phase $\phi_{\mathrm{I}}(n)$ by using

$$
\phi_{\mathrm{I}}(n)=W^{-1}\left\{\psi_{L}(n)\right\}-W^{-1}\left\{\psi_{U}(n)\right\}
$$

However, if the initial value of the unwrapped interferometric phase $\phi_{\mathrm{I}}(1)$ is ambiguous, a constant phase remains to be determined before converting $\phi_{\mathrm{I}}(n)$ into $\varphi_{\mathrm{I}}(n)$. This offset is the difference between the actual absolute interferometric phase value in a certain frame $n_{0}, \varphi_{\mathrm{I}}\left(n_{0}\right)$, and the value of the unwrapped interferometric phase in the same frame, $\phi_{\mathrm{I}}\left(n_{0}\right)$. Therefore $\varphi_{\mathrm{I}}(n)$ can be obtained by using

$$
\varphi_{\mathrm{I}}(n)=\phi_{\mathrm{I}}(n)-\phi_{\mathrm{I}}\left(n_{0}\right)+\varphi_{\mathrm{I}}\left(n_{0}\right)
$$

The actual absolute interferometric phase value in the frame $n_{0}$ may be obtained using prior knowledge of the target position or can be estimated by using some of the absolute phase estimation methods mentioned in [16]. In the implemented processing chain, we are able to track a single prominent scatter, and we assume that we know the actual position of the target in a certain frame.

This unwrapping technique can be unambiguously applied if and only if the phase gradient of the prominent target from frame to frame is less than $\pi$ in each antenna. That is, in a time period of $M / \mathrm{PRF}$, the phase of the scatter must change by less than $\pi$. Typically, we use $N=32$, $M=1$, and a pulse repetition frequency (PRF) of $2000 \mathrm{~Hz}$ in our measurements. With a central frequency of $34 \mathrm{GHz}$, this allows for a relative radial velocity of a prominent scatter from frame to frame of approximately $15.8 \mathrm{~km} / \mathrm{h}$. Although this is adequate for a wide range of applications, this margin could be increased by using a higher PRF.

\subsection{Phase estimation}

The proposed signal processing chain has precision errors in the phase estimation, which are related to the use of the FFT to obtain the range-Doppler ISAR image. As will be shown, these errors are negligible when there is no cell migration in the target detection cell. However, in a real scenario, cell migration may occur from frame to frame in both the range and Doppler directions.

The FFT gives us a sampled version of the actual spectrum of a signal. Depending on the sampling period, we will obtain better accuracy not only in the frequency estimation, but also in the initial phase estimation.

Let us consider a sinusoidal signal of frequency $f_{0}$ with initial phase $\phi_{0}$ sampled with a sampling frequency $f_{\mathrm{s}}$ over an observation time $T_{0}$. The actual spectrum of this signal
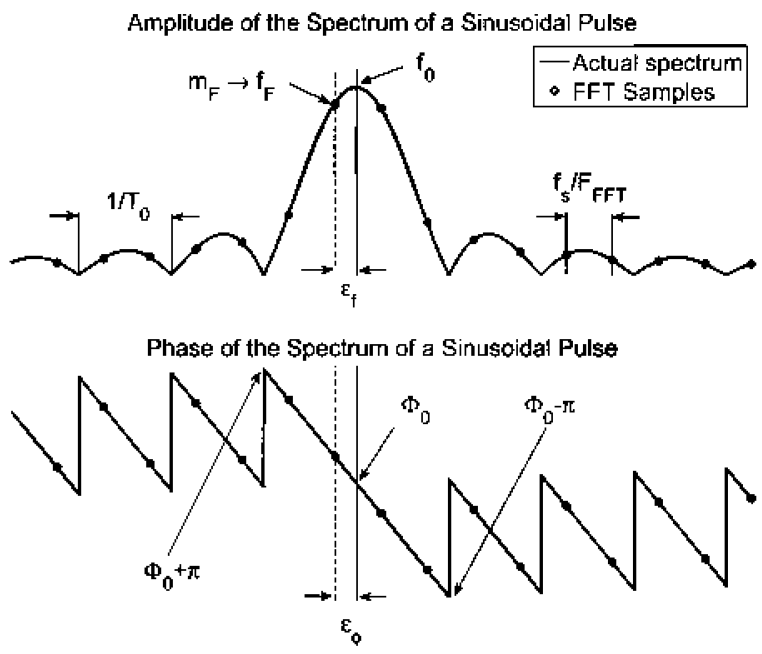

Fig. 4 Phase estimation errors related to the use of the FFT to estimate the actual spectrum

is a sinc signal centred at $f_{0}$ with an absolute phase that varies linearly from $\varphi_{0}+\pi$ to $\varphi_{0}-\pi$ in the main lobe as can be seen in Fig. 4. The main lobe is between the first nulls of the chirp signals and has a width of $2 / T_{0}$. When the sampled spectrum is obtained, the frequency estimation error $\varepsilon_{\mathrm{f}}$ is given by (11), and the phase estimation error $\varepsilon_{\varphi}$ is given by (12). These errors depend on the relative position of the actual frequency, $f_{\mathrm{o}}$, and the frequency bin of the nearest sample of the FFT, $f_{\mathrm{F}}$. The total number of bins in the FFT is denoted by $F_{\mathrm{FFT}}$, and the detected frequency bin number is denoted by $m_{\mathrm{F}}$

$$
\begin{aligned}
\varepsilon_{\mathrm{f}} & =f_{0}-f_{\mathrm{F}} \\
& =f_{0}-\frac{m_{\mathrm{F}} f_{\mathrm{s}}}{F_{\mathrm{FFT}}} \\
\varepsilon_{\varphi} & =-\pi\left(f_{0}-f_{\mathrm{F}}\right) T_{0} \\
& =-\pi\left(f_{0}-\frac{m_{\mathrm{F}} f_{\mathrm{s}}}{F_{\mathrm{FFT}}}\right) T_{0}
\end{aligned}
$$

These simple results can be used to obtain the phase error in the range $\varepsilon_{R}$ and Doppler dimension $\varepsilon_{D}$.

In the range dimension, the frequency of the signal will be the target beat frequency $f_{\mathrm{b}}$, the sampling frequency will be the time sampling frequency $f_{\mathrm{s}}$, the detected beat frequency will be $f_{\mathrm{R}}$, the corresponding bin number will be $m_{\mathrm{R}}$, the total number of bins in the FFT will be $R_{\mathrm{FFT}}$ and the observation time will be the ramp duration, $T_{x}$

$$
\begin{aligned}
\varepsilon_{\mathrm{R}} & =-\pi\left(f_{\mathrm{b}}-f_{\mathrm{R}}\right) T_{x} \\
& =\pi\left(\frac{2 B W}{T_{x} c} R+\frac{m_{\mathrm{R}} f_{\mathrm{s}}}{R_{\mathrm{FFT}}}\right) T_{x}
\end{aligned}
$$

In the Doppler dimension, the frequency of the signal will be the Doppler frequency $f_{\mathrm{d}}$, the sampling frequency will be the pulse repetition frequency $P R F$, the detected Doppler frequency will be $f_{\mathrm{D}}$, the corresponding bin number will be $m_{\mathrm{D}}$, the total number of bins in the FFT will be $D_{\mathrm{FFT}}$ and 
the observation time will be the frame duration, N/PRF

$$
\begin{aligned}
\varepsilon_{\mathrm{D}} & =-\pi\left(f_{\mathrm{d}}-f_{\mathrm{D}}\right) \frac{N}{\mathrm{PRF}} \\
& =\pi\left(\frac{2 v_{\mathrm{R}}}{\lambda}+\frac{m_{\mathrm{D}} \mathrm{PRF}}{D_{\mathrm{FFT}}}\right) \frac{N}{\mathrm{PRF}}
\end{aligned}
$$

where $v_{\mathrm{R}}$ is the target radial velocity.

The measured phase in each frame will be composed of the actual phase plus the errors in each dimension

$$
\phi_{\mathrm{m}}(n)=\phi(n)+\varepsilon_{\mathrm{R}}(n)+\varepsilon_{\mathrm{D}}(n)
$$

It can be seen that the measured phase gradient from frame to frame in (16), $\Delta \phi_{\mathrm{m}}(n)$, is composed of the actual phase gradient, $\Delta \phi(n)$, plus the gradient of the phase errors in each dimension, $\Delta \varepsilon_{\mathrm{R}}(n)$ and $\Delta \varepsilon_{\mathrm{D}}(n)$. Expressions for the gradients of the phase errors can be found in (17) and (18)

$$
\begin{aligned}
\Delta \phi_{\mathrm{m}}(n)= & \phi_{\mathrm{m}}(n+1)-\phi_{\mathrm{m}}(n) \\
= & \phi(n+1)-\phi(n)+\varepsilon_{\mathrm{R}}(n+1)-\varepsilon_{\mathrm{R}}(n) \\
& +\varepsilon_{\mathrm{D}}(n+1)-\varepsilon_{\mathrm{D}}(n) \\
= & \Delta \phi(n)+\Delta \varepsilon_{\mathrm{R}}(n)+\Delta \varepsilon_{\mathrm{D}}(n) \\
\Delta \varepsilon_{\mathrm{R}}(n)= & \varepsilon_{\mathrm{R}}(n+1)-\varepsilon_{\mathrm{R}}(n) \\
= & \pi\left(\frac{2 B W}{T_{x} c} R(n+1)+f_{\mathrm{R}}(n+1)\right) T_{x} \\
& -\pi\left(\frac{2 B W}{T_{x} c} R(n)+f_{\mathrm{R}}(n)\right) T_{x} \\
= & \pi \frac{2 B W}{c} \Delta R(n)+\pi \Delta f_{\mathrm{R}}(n) T_{x} \\
= & \pi \frac{2 B W}{c} \frac{M v_{\mathrm{R}}(n)}{P R F}+\pi \frac{\Delta m_{\mathrm{R}}(n) f_{\mathrm{s}}}{R_{\mathrm{FFT}}} \frac{\mathrm{DC}}{P R F}
\end{aligned}
$$

where DC is the duty cycle of the frequency swept.

$$
\begin{aligned}
\Delta \varepsilon_{\mathrm{D}}(n)= & \varepsilon_{\mathrm{D}}(n+1)-\varepsilon_{\mathrm{D}}(n) \\
= & \pi\left(\frac{2 v_{\mathrm{R}}(n+1)}{\lambda}+f_{\mathrm{D}}(n+1)\right) \frac{N}{\mathrm{PRF}} \\
& -\pi\left(\frac{2 v_{\mathrm{R}}(n)}{\lambda}+f_{\mathrm{D}}(n)\right) \frac{N}{\mathrm{PRF}} \\
= & \pi \frac{2 N}{\lambda \mathrm{PRF}} \Delta v_{\mathrm{R}}(n)+\pi \Delta f_{\mathrm{D}}(n) \frac{N}{\mathrm{PRF}} \\
= & \pi \frac{2 N}{\lambda \mathrm{PRF}} \frac{M a_{\mathrm{R}}(n)}{\mathrm{PRF}}+\pi \frac{\Delta m_{\mathrm{D}}(n) \mathrm{PRF}}{D_{\mathrm{FFT}}} \frac{N}{\mathrm{PRF}}
\end{aligned}
$$

From $(16)-(18)$, it can be seen that the phase gradient between frames depends on two factors. The first is related to the target radial velocity $v_{\mathrm{R}}(n)$ and the radial acceleration $a_{\mathrm{R}}(n)$, and the second factor is related to the cell migrations in the range dimension $\Delta m_{\mathrm{R}}(n)$ and in the Doppler dimension $\Delta m_{\mathrm{D}}(n)$. Assuming that we have a correct sampling in time $\Delta m_{\mathrm{R}}(n)$ and $\Delta m_{\mathrm{D}}(n)$ can only take two values: 1 if a migration is present and 0 if not. Otherwise, the phase gradient will change more than $\pi$ between consecutive frames and the phase unwrapping algorithm cannot be correctly applied as we stated in Section 3.2.
When the detected target remains in the same cell from frame to frame, the only error term is the one related to the target movement. With the typical system parameters given in Table 1, this error can be considered to be negligible. However, when cell migration is present, the second term is clearly dominant, and a peak in the measured phase gradient will appear. This error term can be reduced by using more bins in the range and Doppler FFTs. However, this will cause more cell migration events and an increase in computational burden.

The positions of the peaks in the phase gradient are known because they appear when a cell migration occurs. On the contrary, the exact phase magnitude of the jump $\Delta \varepsilon_{\mathrm{R}}(n)+\Delta \varepsilon_{\mathrm{D}}(n)$ cannot be known, as it depends on the actual target movement. A simple but efficient strategy to filter those peaks in the measured phase gradient $\Delta \phi_{m}(n)$ of each antenna has been developed, with the assumption of smooth movement of the target from frame to frame. For those frames where a cell migration occurs, the value of its phase gradient is replaced by a linear interpolation from the nearest phase gradient values where no cell migration is present. Then, the filtered phase is recovered by integrating the gradient, and adding the initial phase value to this recovered phase. The unwrapped interferometric phase will be obtained using (9) but applying the phase gradient filtering described above, $F\{\cdot\}$, before subtracting the unwrapped phases as shown in

$$
\phi_{\mathrm{I}}(n)=F\left\{W^{-1}\left\{\psi_{L}(n)\right\}\right\}-F\left\{W^{-1}\left\{\psi_{U}(n)\right\}\right\}
$$

To obtain good behaviour with this technique, the cell must be large enough to avoid a cell migration in each frame. In other case, there would be no correct values of the gradient to use in the filtering technique. This can easily be done by changing the number of bins of the FFTs in each dimension, depending on the speed of the target. The existence of peaks in the phase gradient and the performance of this filtering technique will be shown in the simulation results section.

Another method to recover the unwrapped interferometric phase is to obtain the wrapped interferometric phase and then unwrap it. The main reason to unwrap each phase before obtaining the interferometric phase is to be able to apply the gradient filtering separately. Filtering the phase gradient of each antenna is preferable because fewer peaks would be present, just the ones related to the cell migrations of the corresponding antenna, and the filtering would work

Table 1 Parameters used for the simulations and the real experiment

\begin{tabular}{lccc}
\hline Parameters & Symbol & Value & Units \\
\hline central Frequency & $f_{\mathrm{c}}$ & 34 & $\mathrm{GHz}$ \\
RF bandwidth & $\mathrm{BW}$ & 1600 & $\mathrm{MHz}$ \\
pulse repetition frequency & $\mathrm{PRF}$ & 2000 & $\mathrm{~Hz}$ \\
sampling frequency & $f_{\mathrm{s}}$ & 12 & $\mathrm{MHz}$ \\
duty cycle & $\mathrm{DC}$ & 90 & $\%$ \\
baseline length & $B$ & 77 & $\mathrm{~cm}$ \\
baseline inclination & $\alpha$ & 88.4 & $\mathrm{deg}$ \\
central antenna height & $z_{0}$ & 143 & $\mathrm{~cm}$ \\
ramps that form a frame & $N$ & 32 & - \\
ramp slide from frame to frame & $M$ & 1 & - \\
number of bins of the range FFT & $R_{\mathrm{FFT}}$ & 16384 & - \\
number of bins of the Doppler FFT & $D_{\mathrm{FFT}}$ & 128 & - \\
\hline
\end{tabular}


better. The method proposed in [10] is similar to our method but without addressing the phase jump issue because of detection cell migration.

\section{Simulation results}

In order to illustrate and assess the processing chain, a scenario containing an ideal moving point target was simulated. This scenario simulates the one that will be used in the real experiment. In the experiment, a person will start walking towards the radar and simultaneously changing the height of a corner reflector until it reaches the floor. The person will initially accelerate and reach a constant velocity after a certain time, so we will assume a constantly decreasing acceleration in the first $0.5 \mathrm{~s}$. The same reasoning is applied to the last $0.5 \mathrm{~s}$, resulting in a constantly increasing deceleration. Since a similar scenario is used for the simulations and the experiments, the results may allow us to validate our experiment.

For the simulations, the initial position of the target was $x_{T}=0 \mathrm{~m}, y_{T}=50.6 \mathrm{~m}$ and $z_{T}=1.6 \mathrm{~m}$. The target started moving in the first ramp with an acceleration of $-7.2 \mathrm{~m} / \mathrm{s}^{2}$. Over the first $0.5 \mathrm{~s}$, the magnitude of this acceleration decreased linearly, resulting in a velocity of $-1.8 \mathrm{~m} / \mathrm{s}$ and no acceleration. Over the next $2.2 \mathrm{~s}$, the target continued with constant velocity. In the last $0.5 \mathrm{~s}$, the target decelerated at the inverse of the rate in the first $0.5 \mathrm{~s}$, ending with a velocity of $0 \mathrm{~m} / \mathrm{s}$. During the whole time, the height of the target was constantly decreasing at a velocity of $-0.45 \mathrm{~m} / \mathrm{s}$.

Table 1 shows the parameters of the radar and the parameters used to form the ISAR image frames in both the simulations and the real experiment.

To illustrate and validate the expressions given for the gradient peaks in the previous section, a simulation of an ideal point target, moving as described above, was carried out. Fig. 5 shows the range and Doppler cell where the simulated point target is detected from frame to frame, and the phase gradient of the unwrapped phase obtained in the lower antenna. Any cell migration produces a peak in the phase gradient. The magnitude of the phase jump produced by cell migration can be calculated using the second terms of (17) and (18). This yields $1.0354 \mathrm{rad}$ when the cell migration is in the range direction and $0.7854 \mathrm{rad}$ when the cell migration is in the Doppler dimension. These results

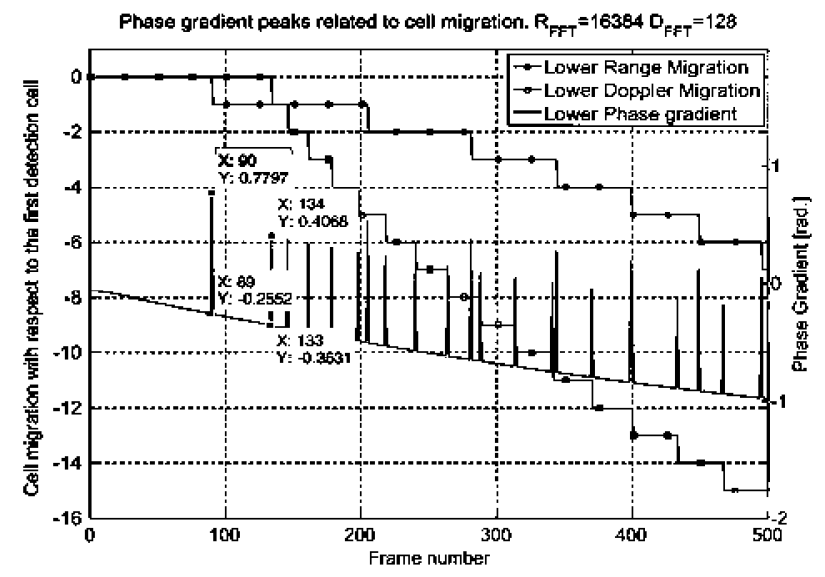

Fig. 5 Peaks that appear in the unwrapped phase gradient because of cell migration in the range and Doppler dimensions, for the lower receiving antenna

The same happens in the upper antenna were very close to the values obtained in the simulations, which can be seen in Fig. 5. Discrepancies in the second or third decimal are due to the terms related to the target velocity and acceleration. The same effect could be observed in the upper antenna as well. Every phase gradient peak will produce a step in the interferometric phase, which is removed with the gradient filtering method (Fig. 8).

The effect of increasing the number of bins in the range FFT and in the Doppler FFT are shown in Fig. 6. As stated in Section 3.3, it is clearly shown that the magnitude of the phase jumps are reduced and the number of jumps is increased.

As previously stated, increasing the system resolution will reduce the probability of different scatters falling in the same resolution cell. However, multipath echoes could always be present in a real environment. In order to simulate the multipath effect, the reflections from the floor are simulated for both the transmitting and receiving antennas and coherently added to the direct path signals. It is interesting to compare the results of the simulations with and without the multipath echoes.

It is well known that the multipath phenomenon cause a ripple in the received power. In Fig. 7, the received power in each antenna is shown. When multiple scattering centres are detected in the same resolution cell, the resultant phase will suffer from scintillation. A similar effect is observed when echoes of the same target, with different propagation paths, arrive in the same resolution cell. As a consequence, the height measurement will be affected. The effect of multipath echoes on the height measurement is shown in Fig. 8. When multipath echoes were not present, the measured height was quite accurate, but a ripple around the actual height appeared when the multipath echoes were present. The maximum deviation was around $10 \mathrm{~cm}$. In any
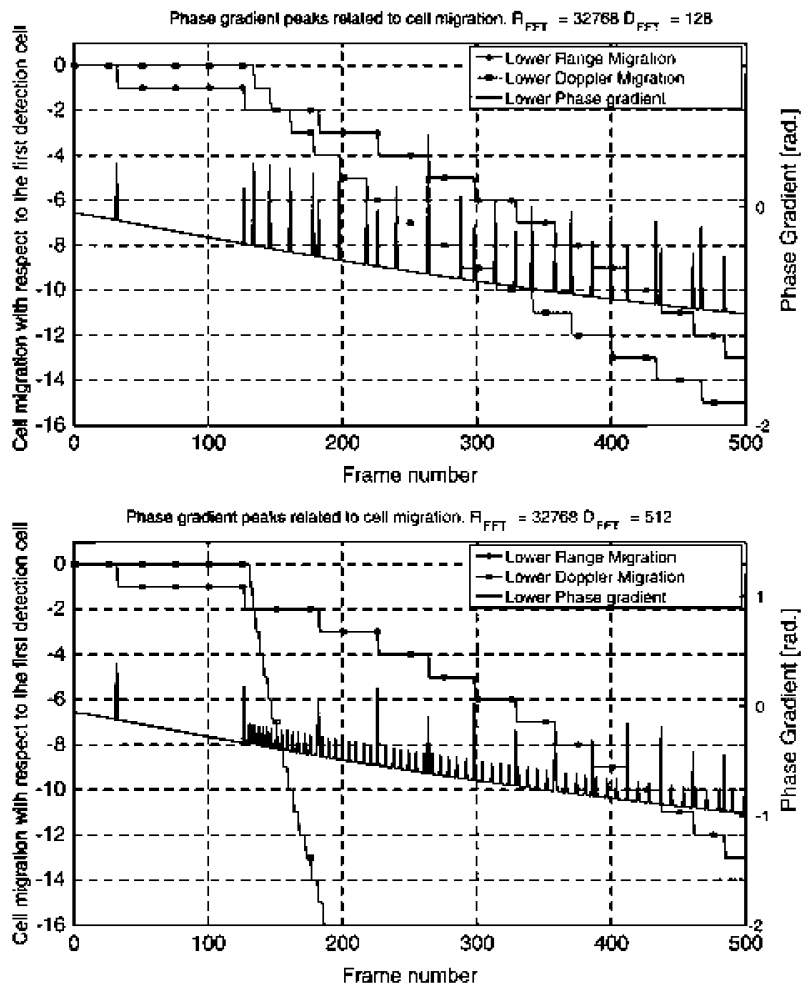

Fig. 6 Effect of increasing the FFT bins in the phase gradient

The phase gradient where no cell migration is present remains unchanged but the magnitudes of the peaks, in each dimension, is reduced. More peaks appear because of the increase in cell migration 


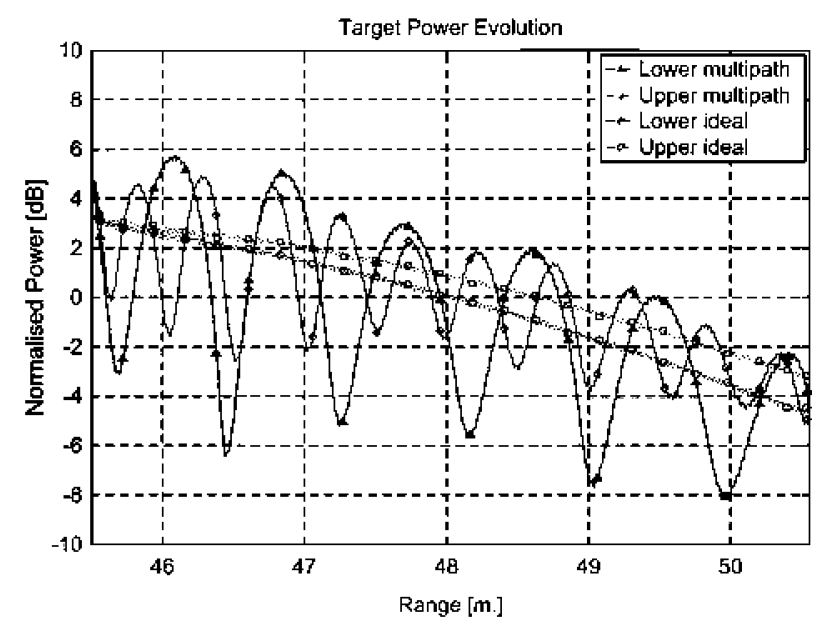

Fig. 7 Effect of multipath on the received power in each antenna for simulated data

case, it can be stated that the whole processing chain was correct, and that the actual height was tracked quite well.

Also shown in Fig. 8 is a comparison between the heights obtained with and without phase gradient filtering when multipath echoes are present. It can be seen that the filtering method definitively improved the quality of the measured height. When no filtering was applied, the recovered height was full of discontinuities caused by cell migration, as explained before. A drift may also appear if the number of positive and negative jumps are not balanced. However, they are usually balanced, because when a cell migration occurs in one of the antennas, the same migration will occur in the other antenna in the next few frames. As the interferometric phase of each antenna is obtained by subtracting both phases as in (9), no long-term drift appears.

In Fig. 9, the evolution of the detected speed of the target is shown. No significant effect of multipath phenomenon was observed. Finally, Fig. 10 presents a three-dimensional representation of the target, tracked in the range, speed and height axes.

\section{Experimental results}

In the real experiment, a person started walking towards the radar and simultaneously changing the height of a corner

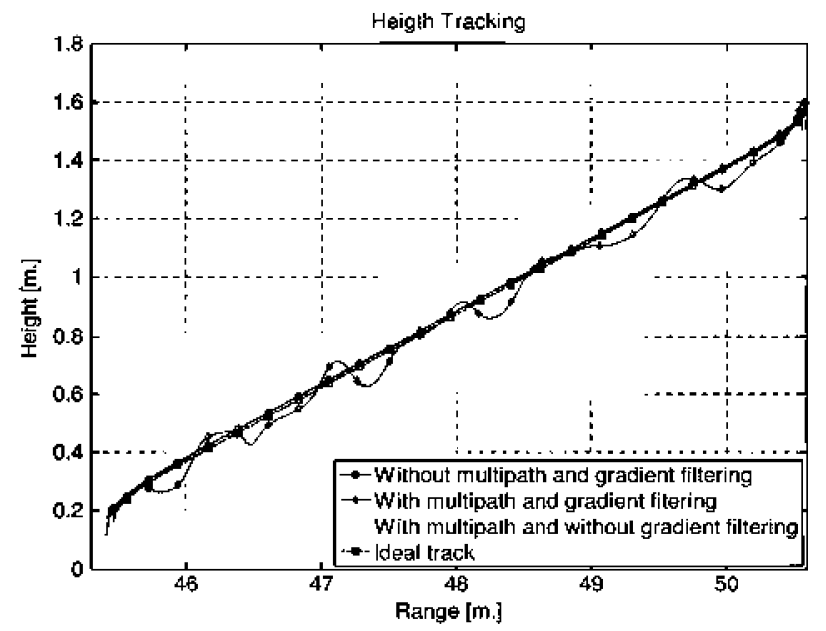

Fig. 8 Effect of multipath on the height estimation for simulated data

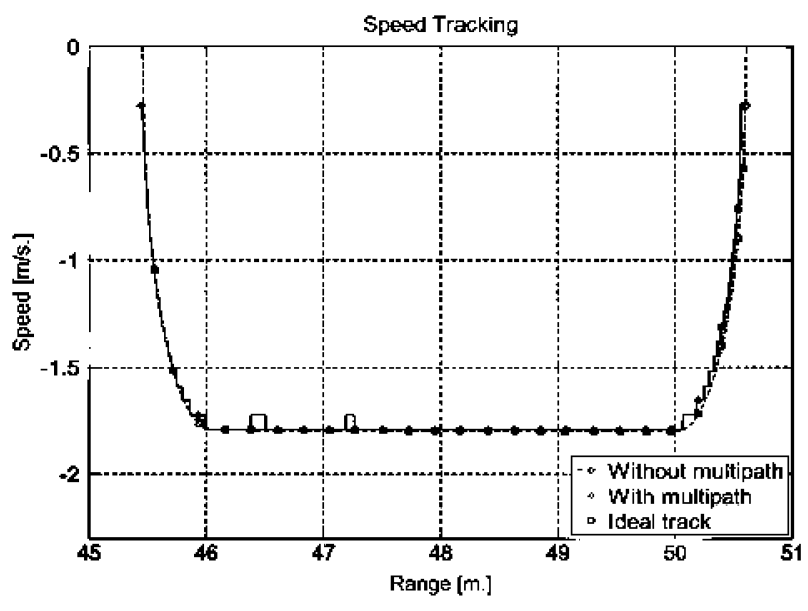

Fig.9 Effect of multipath on the speed estimation for simulated data

reflector until it reached the floor. This person tried to walk with a constant velocity. The person started moving just after the radar had started measuring, and the radar was stopped just after the target had reached the ground. This was done to ensure that the initial and final accelerations of the target were captured. The experiment was conducted indoors, with the person walking along a corridor. The

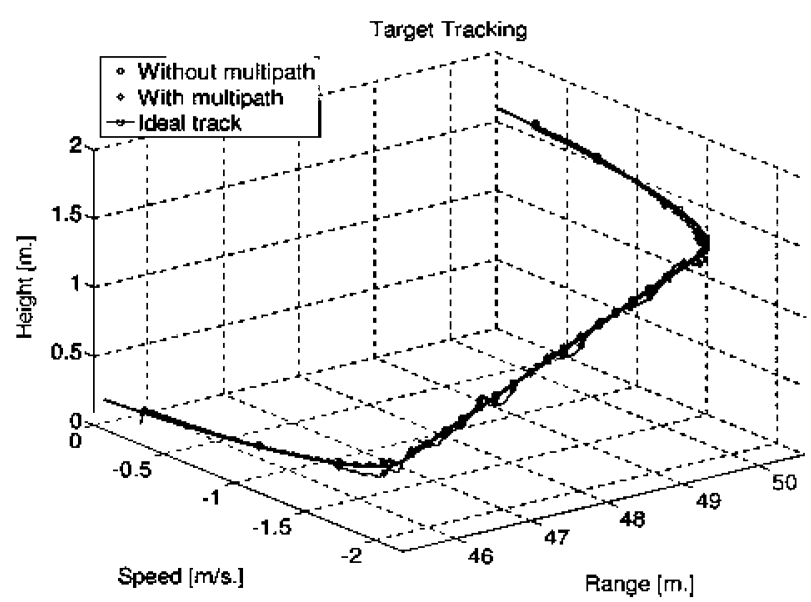

Fig. 10 Effect of multipath on the trajectory estimation for simulated data

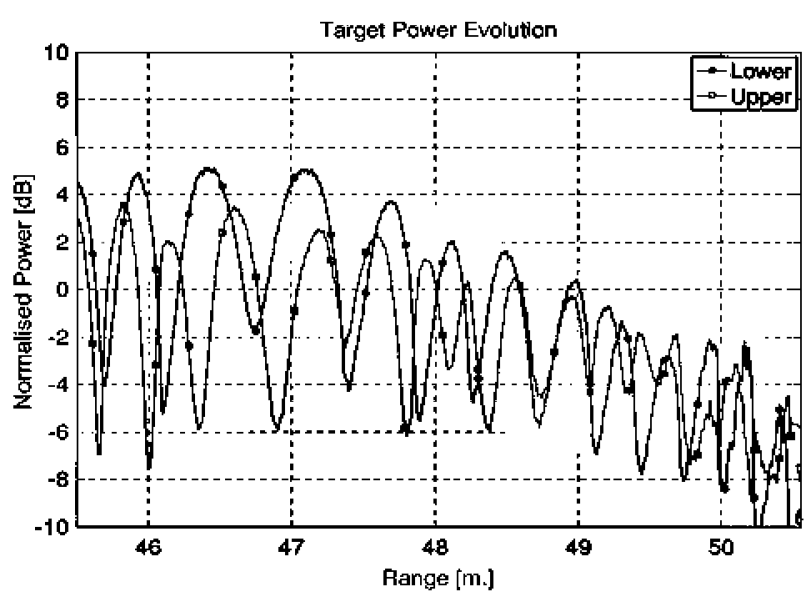

Fig. 11 Received power in each antenna in the real experiment 


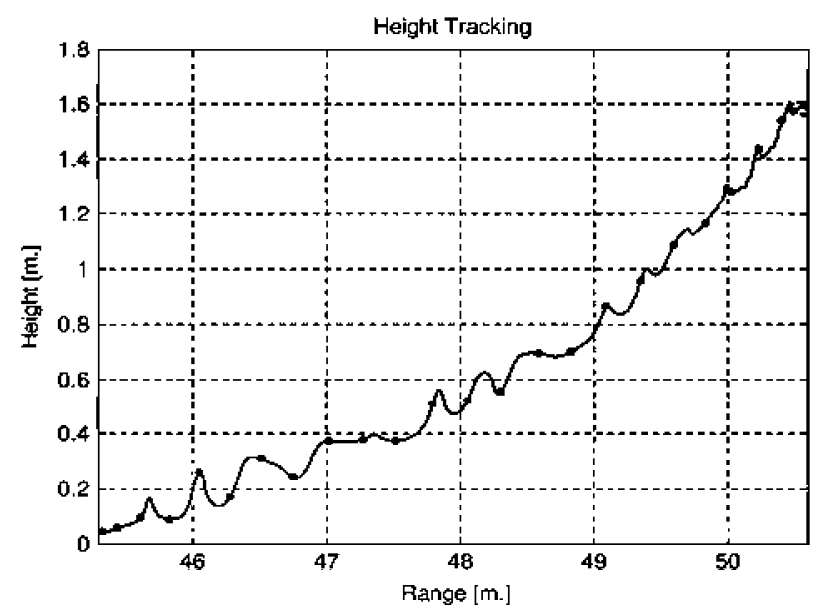

Fig. 12 Height estimation in the real experiment

parameters of the radar were those shown in Table 1. The initial position of the target was the same position that was used in the simulations, but the speed of the person may have differed slightly. As the experiment was conducted indoors, we expected a strong multipath echoes influence. This hypothesis is confirmed with the received powers in each antenna as functions of the distance to the radar, which are shown in Fig. 11. The height measurement is shown in Fig. 12. It can be stated that a clear multipath

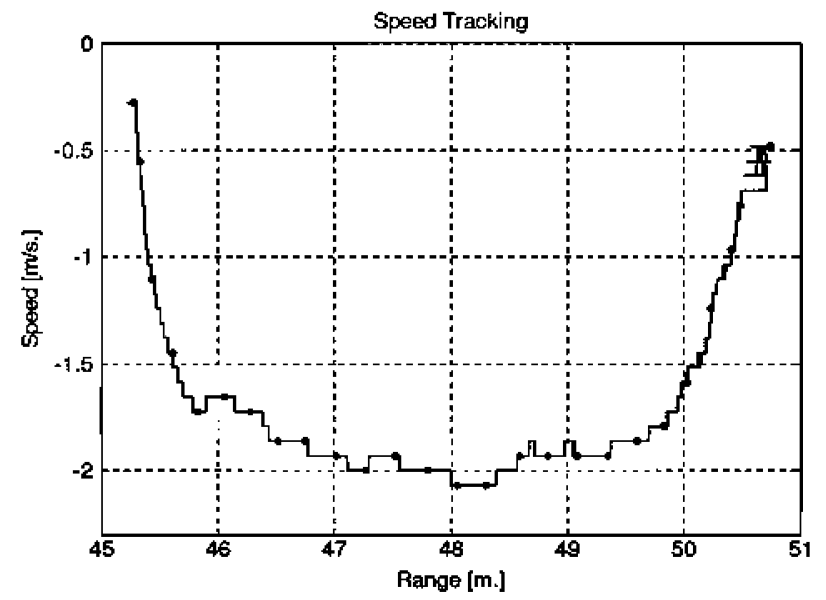

Fig. 13 Speed estimation in the real experiment

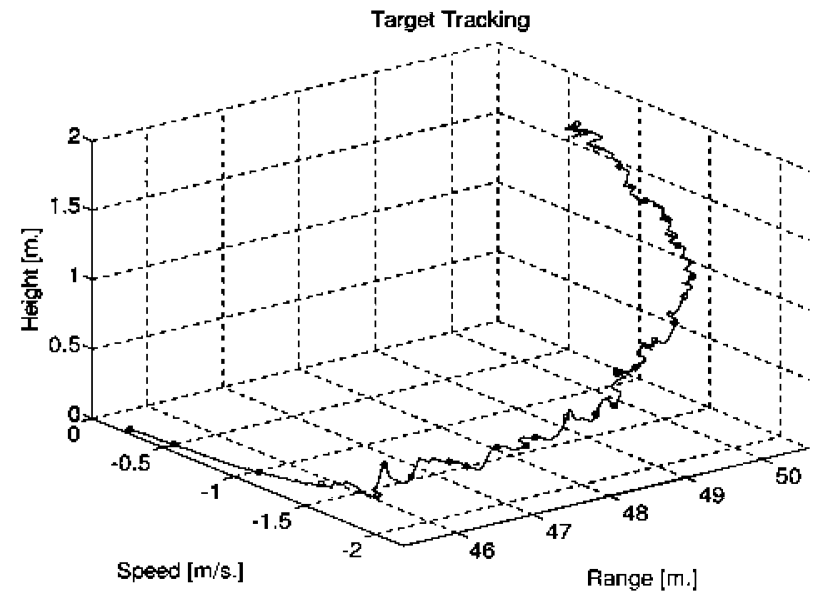

Fig. 14 Trajectory estimation in the real experiment effect is shown in both the power and height measurements, with a similar ripple to that observed in simulations. The speed measurement in Fig. 13 and the three-dimensional representation of the measured quantities in Fig. 14 are quite similar to the ones obtained in the simulations, which supports the experimental results.

\section{Conclusions}

The range, speed and height of a moving target have been correctly tracked in consecutive ISAR image frames, verifying the performance of the experimental prototype. These results are a key step towards demonstrating the feasibility of using this interferometric LFMCW millimetre-wave radar for InISAR applications. Furthermore, a promising phase unwrapping technique has been presented and tested on simulated and real data. The influence of multipath echoes in the InISAR height measurement has also been studied.

In the implemented processing chain, we are capable of tracking a single prominent scatter with the assumption that we know the actual position of the target in a certain frame. Further work is being carried out in order to solve these limitations so that the system can track several prominent points in the same ISAR image from frame to frame and determine the correct height without prior knowledge of the target position.

\section{Acknowledgments}

This work has been supported by the projects TEC200507010-C02 and TEC2008-02148 of the Spanish National Board of Scientific and Technology Research.

\section{References}

1 Xiaojian, X., Narayanan, R.M.: 'Three-dimensional interferometric ISAR imaging for target scattering diagnosis and modelling', IEEE Trans. Image Process., 2001, 10, (7), pp. 1094-1102

2 Xiaojian, X., Hong, L., Peikang, H.: '3-D interferometric ISAR images for scattering diagnosis of complex radar targets'. IEEE Radar Conf., Waltham, MA, USA, April 1999, pp. 237-241

3 Xiaojian, X., Narayanan, R.M.: 'Enhanced resolution in 3-D interferometric ISAR imaging using an iterative SVA procedure', Proc. Geosci. Remote Sens. Symp., 2003, 2, pp. 935-937

4 Changzheng, M., Tat, S.Y., Qun, Z., Hwee, S.T., Jun, W.: 'Threedimensional ISAR imaging based on antenna array', IEEE Trans. Geosci. Remote Sens., 2008, 46, (2), pp. 504-515

5 Liang, H.-Q., He, M.-Y., Li, N.-J., Zhang, L.-X.: 'The research of nearfield InISAR imaging diagnosis'. Int. Conf. on Microwave and Millimeter Wave Technology, Nanjing, China, April 2008, pp. 1773-1775

6 Qun, Z., Tat, S.Y., Gan, D., Shouhong, Z.: 'Estimation of threedimensional motion parameters in interferometric ISAR imaging', IEEE Trans. Geosci. Remote Sens., 2004, 42, (2), pp. 292-300

7 Mayhan, J.T., Burrows, M.L., Cuomo, K.M., Piou, J.E.: 'High resolution 3D 'Snapshot' ISAR imaging and feature extraction', IEEE Trans. Aerosp. Electron. Syst., 2001, 37, (2), pp. 630-642

8 Qun, Z., Tat, S.Y.: 'Three-dimensional SAR imaging of a ground moving target using the InISAR technique', IEEE Trans. Geosci. Remote Sens., 2004, 42, (9), pp. 1818-1828

9 Qun, Z., Tat, S.Y.: 'Novel registration technique for InISAR and InSAR'. Proc. Geoscience Remote Sensing Symp., 2003, vol. 1, pp. 206-208

10 James, A.G., William, R.S.: 'Generalized ISAR - Part II: interferometric techniques for three-dimensional location of scatterers', IEEE Trans. Image Process., 2005, 14, (11), pp. 1792-1797

11 Soumekh, M.: 'Automatic aircraft landing using interferometric inverse synthetic aperture radar imaging'. Int. Conf. on Image Processing, Washington, DC, USA, 1995, vol. 1, pp. 129-132

12 Bertl, S., Dallinger, A., Detlefsen, J.: 'Broadband circular interferometric millimeter-wave ISAR for threat detection', Adv. Radio Sci., 2007, 5, pp. $147-151$ 
13 Smith, B.J., Rock, J.C., McFarlin, S.: 'A synthetic interferometric ISAR technique for developing 3-D signatures', IEEE Proc. Aerosp. Conf., 2003, 2, pp. 1055-1065

14 Liya, L., Weiming, Y., Hongwei, L., Bo, C., Shunjun, W.: 'Radar automatic target recognition based on InISAR images'. First Asian and Pacific Conf. on Synthetic Aperture Radar, 2007, pp. $497-502$

15 Skolnik, M.I.: 'The radar equation' in Skolnik, M.I. (Ed.): 'Introduction to radar systems' (McGraw-Hill, 1980, 2nd edn.), pp. 52-53
16 Rosen, P.A., Hensley, S., Joughin, I.R., et al: 'Synthetic aperture radar interferometry', Proc IEEE, 2000, 88, (3), pp. 333-382

17 Mark, D.P., Dennis, C.G.: 'Two-dimensional phase unwrapping: theory, algorithms, and software' (John Wiley and sons, 1998)

18 Almorox-Gonzalez, P., Gonzalez-Partida, J.T., Burgos-Garcia, M., de la Morena-Alvarez-Palencia, C., Arche-Andradas, L., Dorta-Naranjo, B.P.: 'Portable high resolution LFM-CW radar sensor in millimeter-wave band'. Int. Conf. on Sensor Technologies and Applications, 2007, pp. 5-9 